\title{
Peripheral Merve Stimulation of the Lateral Femoral Cutaneous and Superior Gluteal Merves for Treating Proximal Neuropathy Hip Pain
}

\author{
Matthias Wiederholz, MD' \\ Alaa Abd-Elsayed, MD² \\ Niek Vanquathem, $\mathrm{BA}^{3}$
}

Background: Chronic postoperative hip pain is estimated to occur in $10 \%$ to $35 \%$ of patients undergoing total hip replacement. Proximal peripheral neuropathic pain of the lateral femoral cutaneous and superior gluteal nerves has proven to be a difficult disorder to treat. Opioids are often ineffective in the treatment of neuropathic pain. Interventional methods such as peripheral nerve stimulation are minimally invasive options capable of relieving neuropathic pain. Stimulators powered by an implantable pulse generator (IPG), however, may not be suitable for peripheral nerve stimulation because of difficulty finding an appropriate pocket site. The introduction of wireless peripheral nerve stimulation has improved the ability to offer this modality.

Case

Presentation: We present a case of proximal peripheral neuropathic pain of the lateral femoral cutaneous and superior gluteal nerves that failed all other treatment modalities including spinal cord stimulation and intrathecal drug delivery. Two quadripolar, tined, wireless electrode arrays were positioned over the lateral femoral cutaneous and superior gluteal nerves. A stimulation scheme with a pulse rate of $1.5 \mathrm{kHz}$ and pulse width of $30 \mu \mathrm{s}$ at $2.0 \mathrm{~mA}$ was tested and found effective.

Conclusion: This patient had proximal neuropathic hip pain and failed a variety of chronic pain treatment options, including conventional IPG-based spinal cord stimulation and an intrathecal drug delivery system. She was successfully treated with a wireless peripheral nerve stimulation system.

Key words: Hip pain, lateral femoral cutaneous nerve, peripheral nerve stimulator, peripheral neuropathy, superior gluteal nerve

\footnotetext{
From: ${ }^{1}$ Performance Pain and Sports Medicine, Houston, TX; ${ }^{2}$ Wisconsin School of Medicine and Public Health, Madison, Wl; ${ }^{3}$ Stimwave Technologies, Inc., Pompano Beach, FL

Corresponding Author: Niek Vanquathem, BA E-mail: niekv@stimwavefreedom.com 


\section{BACKGROUND}

The prevalence of chronic pain when walking following total hip arthroplasty (THA) can be as high as $35 \%$ (1), limiting daily activities. Chronic hip pain is multifactorial and includes neuropathic (2), nociceptive (3), and psychosocial components (4). This makes the diagnosis very challenging. Pain following THA is often described as neuropathic burning in the trochanteric area with allodynia (5). It can also present with nonspecific symptoms that may be confused with various other pain origins, intraarticular or extraarticular. In addition, neuropathy of nerves innervating the hip region can cause radiating pain in an extended region of the trunk, buttocks, and upper leg, which can further complicate an accurate diagnosis $(6,7)$.

Successful management of hip pain depends on accurately determining the origin of the pathology. Certain patients may require surgical interventions, while others can generally be treated with medication or less invasive options in the initial stages of degeneration. Chronic hip pain can be resistant to conservative medical management treatment, including physical therapy, medications, and injections. Peripheral nerve (PNS) and peripheral field nerve stimulation (PFNS) have been used to treat pain of peripheral nerve origin for a variety of neuropathic conditions (8) including postherpetic (9), craniofacial (10-17), ilioingual (18), and intercostal (19) neuropathic pain.

It can be difficult to determine whether hip pain originates from nerves within the joint itself, or from nerve pathology in surrounding nerve tissue. Precision-guided injections of the intraarticular joint space as well as the surrounding innervating nerves can help to differentiate the cause. If the pain is confirmed by injections to be due to a particular nerve, and the patient notes only temporary relief, PNS may be a treatment option. The use of wireless PNS to treat hip pain is a novel use of this technology.

\section{CASE PRESENTATION}

The patient in this case is a 76-year-old woman with a history of spinal fusion, with 14 spinal screws placed 10 years after the spinal fusion. She had also undergone a right hip arthropathy (THA), a right hip tendon split one year after hip replacement, and a second right hip replacement 14 years after the original. A conventional spinal cord stimulator (SCS) and an intrathecal pump implant kept most of her back pain under control, but she was still experiencing severe left hip pain and was not considered a candidate for another hip surgery. She was wheelchair-bound due to the immobilizing pain. The patient presented with severe pain in the back of the left hip (consistent with superior gluteal neuralgia) as well as in the anterolateral aspect of the thigh in a classic pattern of meralgia paresthetica. After a series of successful, but short-lived lateral femoral cutaneous nerve (LFCN) and superior gluteal nerve (SGN) injections, we diagnosed the patient with neuropathy of the LFCN and SGN.

\section{Surgical Description}

Trial: The patient was positioned on her side to grant full access to her left hip. She was sterilely prepped and draped. Fluoroscopy and landmarks were used to plan the introducer entry point and route for 28 8-contact wireless trial electrode arrays (Stimwave, Pompano Beach, $\mathrm{FL}$ ), one targeting the left LFCN and the second targeting the left SGN. The introducer entry points were injected with local anesthetic. A 13-gauge (G) introducer was used to enter the skin at the left anterior superior iliac spine (ASIS) and was directed horizontally toward the umbilicus. The trial electrode array was inserted through the introducer toward the LFCN target. The electrodes were confirmed to be at the left LFCN (Fig. 1) with fluoroscopy confirming placement near bony landmarks.

A second 13-G introducer was used to enter the skin just superior and posterior to the greater trochanter and placed inferiorly towards the knee. The second trial electrode array was inserted through the introducer and placement was confirmed with fluoroscopy to be at the SGN (Fig. 2). Both introducers were removed, steering stylets retracted and receivers were insterted into the inner lumen of the electrode arrays. Intraoperative testing was performed with a trial transmitter placed in a sterile bag. Coverage of the painful areas was confirmed. The trial electrode arrays were then secured with Mastisol (Ferndale Laboratories, Ferndale, MI) and Steri-Strips (3M, Saint Paul, MN) and completely covered under a sterile Tegaderm (3M, Saint Paul, MN). The patient completed a 6-day trial and reported $80 \%$ pain relief.

Permanent Implant: The placement techniques for the electrode arrays utilized in the trial were repeated for the LFCN and SGN utilizing 2 4-contact, tined, permanent electrode arrays with separate receiver devices. The receivers were inserted into the inner lumen of the electrode arrays. The introducers were removed. A receiver pocket was created on the patient's hip distal 


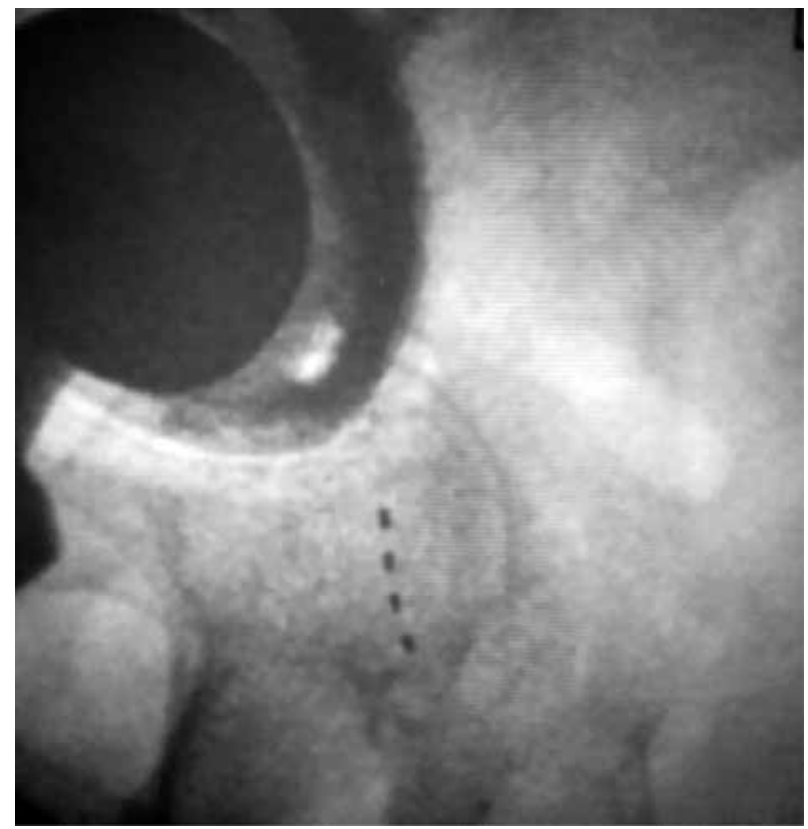

Fig. 1. Image showing the quadripolar neurostimulator electrode array at the lateral femoral cutaneous nerve (LFCN)

to the last (cut) marker bands. The introducer was then used to tunnel the SGN receiver neurostimulator straight upward towards the hip, completely burying the receiver under the skin, leaving the tail in the receiver pocket. An anchor stitch was placed through the fascia and around the body of the LFCN receiver at the device proximal marker band to facilitate a 90-degree upward bend; the stimulator was tunneled to the same receiver pocket as the first receiver. Intraoperative testing was performed and coverage of the painful areas was confirmed. A knot was tied to permanently mate the receivers and electrode arrays. The distal portion of the stimulators were coiled, sutured to itself to eliminate any sharp ends, and then sutured to the fascia. The pocket was closed with subcutaneous and then subcuticular sutures.

The patient tolerated the procedure very well. A stimulation scheme with a pulse rate of $1.5 \mathrm{kHz}$ and pulse width of $30 \mu \mathrm{s}$ at $2.0 \mathrm{~mA}$ was tested and found effective. The patient experienced $80 \%$ improvement in Visual Analog Scale (VAS) pain scores from baseline after the implantation and continued to have $80 \%$ improvement at 1, 3, and 6 months' follow-up.

\section{DISCUSSION}

The LFCN arises from the lumbar plexus. It provides

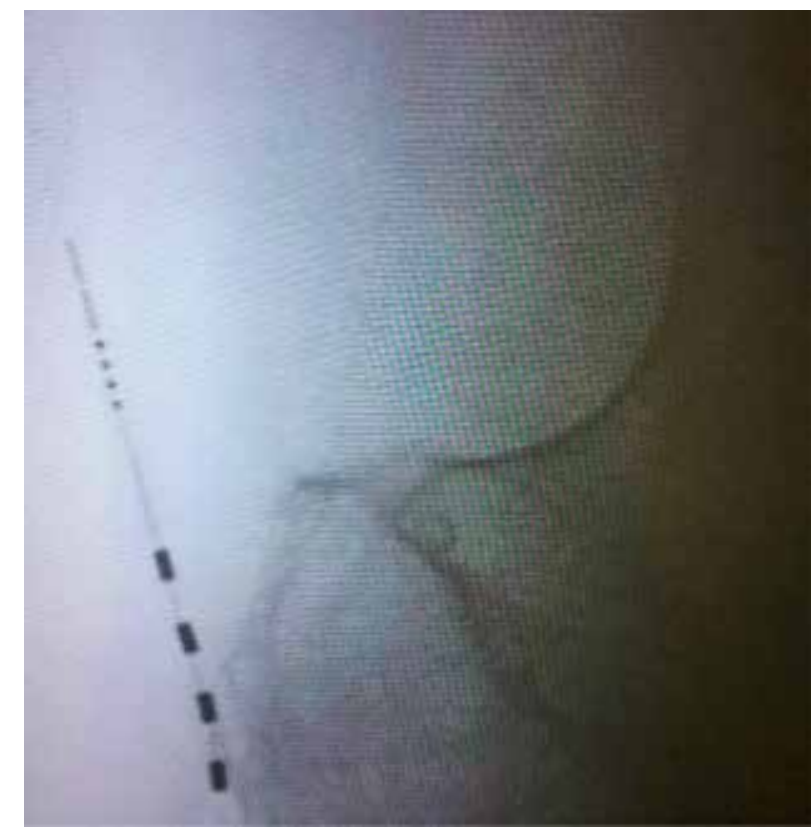

Fig. 2. Image showing the quadripolar neurostimulator electrode array at the superior gluteal nerves

sensory innervation to the anterolateral thigh area. Entrapment of the nerve causes meralgia paresthetica, which can present as hip pain radiating to the anterolateral aspect of the thigh. The nerve can be affected by various factors including entrapment anywhere along its course, trauma, and additional damage from surgical interventions $(20,21)$. The SGN originates from the sacral plexus. It lies between the piriformis and gluteus medius muscles and innervates the gluteus medius and minimus muscles. The nerve passes about 5 centimeters superior to the tip of the greater trochanter (22).

The anatomical course of both nerves, in close proximity to the hip, can make diagnosis of pain in the region challenging. Nerve blocks are generally good diagnostic tools in determining whether neuropathy is a factor. Pain in the hip joint region has varying origins, which can be related to the joint itself, muscles and tendons around the joint, and/or neuropathy of nerves in close relation to the joint. In our case, the patient tried several modalities for treating her hip pain with limited success. While nerve blocks did not provide lasting relief, they were successfully utilized to diagnose the cause of the pain. Wireless PNS was able to control pain after the failure of all other treatment options including spinal cord stimulation and intrathecal drug delivery. 


\section{CONCLUSION}

We presented a case of wireless PNS of the lateral femoral cutaneous and superior gluteal nerves causing pain in the hip region with excellent outcomes. PNS should be considered for treating peripheral neuropathies when other conservative measures fail.

\section{REFERENCES}

1. Johnsson $R$, Thorngren KG. Function after total hip replacement for primary osteoarthritis. Int Orthop 1989; 13:221-225.

2. Perkins FM, Kehlet $\mathrm{H}$. Chronic pain as an outcome of surgery. Anaesthesiology 2000; 93:1123-1133.

3. Gwilym SE, Pollard TCB, Carr AJ. Understanding pain in osteoarthritis. J Bone Joint Surg (Br) 2008; 90:280-287.

4. Woolf AD, Pfleger B. Burden of major musculoskeletal conditions, policy and practice. Bull World Health Organ 2003: 81:646-656.

5. Nikolajsen L, Brandsborg B, Lucht U, Jensen TS, Kehlet H. Chronic pain following total hip arthroplasty: A nationwide questionnaire study. Acta Anaesthesiol Scand 2006; 50:495-500.

6. Hickman JM, Peters CL. Hip pain in the young adult: Diagnosis and treatment of disorders of the acetabular labrum and acetabular dysplasia. Am J Orthop (Belle Mead NJ) 2001; 6:459-467.

7. Northmore-Ball MD. Young adults with arthritic hips. BMJ 1997; 7103:265-266.

8. Van Buyten J-P, Van Zundert J, Milbouw G. Treatment of failed back surgery syndrome patients with low back and leg pain: A pilot study of a new dual lead spinal cord stimulation system. Neuromodulation 1999; 2:258-265.

9. Yakovlev A, Peterson A. Peripheral nerve stimulation in treatment of intractable postherpetic neuralgia: A case report. Neuromodulation 2007; 10:373-375.

10. Weiner RL, Garcia CM, Vanquathem N. A novel miniature, wireless neurostimulator in the management of chronic craniofacial pain: Preliminary results from a prospective pilot study. Scand J Pain 2017; 17:350-354.

11. Slavin KV, Nersesyan $H$, Wess $C$. Peripheral neurostimulation for treatment of intractable occipital neuralgia. Neurosurgery 2006; 58:112-119.

12. Johnstone CS, Sundaraj R. Occipital nerve stimulation for the treatment of occipital neuralgia-eight case studies. Neuromodulation 2006; 9:41-47.

13. Oh MY, Ortega J, Bellotte JB, Whiting DM, Aló K. Peripheral nerve stimulation for the treatment of occipital neuralgia and transformed migraine using a C1-2-3 subcutaneous paddle style electrode: A technical report. Neuromodulation 2004; 7:103-112.

14. Weiner RL, Reed KL. Peripheral neurostimulation for control of intractable occipital neuralgia. Neuromodulation 1999; 2:217-221.

15. Johnson MD, Burchiel KJ. Peripheral stimulation for treatment of trigeminal postherpetic neuralgia and trigeminal posttraumatic neuropathic pain: A pilot study. Neurosurgery 2004; 55:135-141.

16. Slavin $\mathrm{KV}$, Wess $\mathrm{C}$. Trigeminal branch stimulation for intractable neuropathic pain: Technical note. Neuromodulation 2005; 8:7-13.

17. Oberoi J, Sampson C, Ross E. Head and neck peripheral stimulation for chronic pain report of 3 cases. Neuromodulation 2008; 11:272-276

18. Stinson LW Jr, Roderer GT, Cross NE, Davis BE. Peripheral subcutaneous electrostimulation for control of intractable post-operative inguinal pain: A case report series. Neuromodulation 2001; 4:99-104.

19. Tamimi MA, Davids HR, Langston MM, Krutsch JP, Yakovlev A, Barolat G. Successful treatment of chronic neuropathic pain with subcutaneous peripheral nerve stimulation. Neuromodulation 2009; 12:210-214.

20. Standring S (ed). Gray's Anatomy: The Anatomical Basis of Clinical Practice. Churchill Livingstone/Elsevier, London, 2008.

21. Witkin LR, Gulati A, Zhang T, Karl HW. Superior gluteal nerve entrapment. In: Trescot A (ed). Lateral femeral cutaneous nerve entrapment. Springer, New York, NY, 2016, pp 667-681.

22. Trescot A. Superior gluteal nerve entrapment. In: Trescot A (ed). Peripheral nerve entrapments: Clinical diagnosis and management. Springer, New York, NY, 2016, pp 571-579. 